\title{
Complicaciones del abordaje endonasal directo transesfenoidal en el manejo de adenomas de hipófisis
}

\author{
J.H. Sandoval-Sánchez; F. Flores-Cárdenas; Mª.C. Vargas-Frutos* y J.M. Páez-Ontiveros
}

Departamentos de Neurocirugía y de Medicina Interna*. Unidad Médica de Alta Especialidad. Hospital de Especialidades. Centro Médico Nacional de Occidente. Instituto Mexicano del Seguro Social. Guadalajara. Jalisco. México.

Resumen

Objetivo. Describir la frecuencia de complicaciones y molestias del abordaje endonasal directo transesfenoidal, utilizado en la resección de adenomas de hipófisis.

Pacientes y métodos. En forma retrospectiva, se revisaron los expedientes de pacientes con adenoma de hipófisis, en quienes se realizó el abordaje endonasal directo transesfenoidal, dentro de un periodo comprendido entre Agosto del 2003 y Diciembre del 2004. Se recolectó información sobre las complicaciones trans y postoperatorias, además de molestias postoperatorias registradas en el expediente.

Resultados. Se incluyeron 41 pacientes, 23 (56.1\%) hombres y 18 (43.9\%) mujeres, con una edad promedio de $47.7 \pm 16.8$ años. Dentro de las complicaciones transoperatorias, tres pacientes $(7.3 \%)$ tuvieron hemorragia intraselar, dos $(4.9 \%)$ hipertensión arterial descontrolada y uno (2.4\%) fístula de líquido cefalorraquídeo. Entre las complicaciones postoperatorias, la más frecuente fue la diabetes insípida con diez casos $(24.4 \%)$, dos $(4.9 \%)$ presentaron insuficiencia hipofisaria anterior y uno (2.4\%) sinusitis. La molestia más común fue la congestión nasal en trece pacientes $(31.7 \%)$.

Conclusiones. El abordaje endonasal directo transesfenoidal tiende a presentar menos complicaciones y molestias especialmente nasales, por lo que en un futuro podría ser considerado como el abordaje preferido para la resección de adenomas de hipófisis.

PALABRAS CLAVE. Adenoma de hipófisis. Tratamiento quirúrgico. Abordaje endonasal directo transesfenoidal. Complicaciones.

Complications of the direct endonasal transsphenoidal approach in the management of pituitary adenomas

Recibido: 1-02-07. Aceptado: 21-03-07
Sumary

Objective. To describe the frequency of complications and complaints caused by direct endonasal transsphenoidal approach used in the resection of pituitary adenomas.

Patients and methods. In a retrospective way, we review patients' records with pituitary adenomas, operated by means of direct endonasal transsphenoidal approach, between August 2003 and December 2004. We recollected information about operative and postoperative complications, besides complaints registered in patients' records.

Results. 41 patients were included, $23(56.1 \%)$ males and $18(43.9 \%)$ females, with a mean age of 47.7 $\pm \mathbf{1 6 . 8}$ years. Operative complications were intrasellar hemorrhage in three patients $(\mathbf{7 . 3 \%})$, hypertension in two (4.9\%) and cerebrospinal fluid fistula in one (2.4\%).

On the other hand, postoperative complications were diabetes insipidus $(\mathbf{2 4 . 4 \% )}$ ) in ten cases, anterior pituitary insufficiency $(4.9 \%)$ in two and sinusitis (2.4\%) in one. Nasal congestion was the most common complaint $(31.7 \%)$ in thirteen patients.

Conclusions. Supported in our results, the direct endonasal transsphenoidal approach tends to present mainly fewer nasal complications and complaints, and in the future it could be considered like the preferred approach for resection of pituitary adenomas.

KEYWORDS. Pituitary adenoma. Surgical treatment. Direct endonasal transsphenoidal approach. Complications.

Introducción

Los adenomas de hipófisis son neoplasias benignas que representan entre el 10 y $15 \%$ de los tumores intracraneales, encontrándoseles incidentalmente hasta en el $20 \%$ de las autopsias $^{2,22}$. Con excepción del prolactinoma, el tratamiento de primera línea para la mayoría de los adenomas, es la resección quirúrgica ${ }^{16}$; la cual puede ser a través del 
cráneo o seno esfenoidal. En el caso de las rutas hacia el seno esfenoidal, se han descrito las vías transantral, transmaxilar, sublabial y endonasal. Las dos primeras rara vez se emplean en la actualidad, mientras que la endonasal transesfenoidal es la ruta preferida para remover más del $90 \%$ de las lesiones de la silla turca ${ }^{5,18}$. Dentro del abordaje endonasal transesfenoidal, existen dos variantes: 1. Endonasal transeptal transesfenoidal (Hirsch, 1910), 2. Endonasal directo transesfenoidal (Griffith y Veerapen, 1987) $)^{10,13,14,17}$.

En el abordaje endonasal transeptal transesfenoidal, se realiza una incisión en la columnela a nivel de la unión de la piel con la mucosa nasal, y con ello una amplia disección de dicha mucosa en el plano subpericondrial ${ }^{5,26}$. Por otra parte, el abordaje endonasal directo transesfenoidal requiere mínima disección de la mucosa nasal, ya que se efectúa una incisión vertical más posterior, esto es, a nivel de la unión del cartílago septal con el septum óseo y a partir de ahí se realiza la disección, para luego luxar el cartílago septal e identificar el vómer, sin embargo, este abordaje provee un trayecto ligeramente fuera de la línea media $^{8,10,18,21,25}$.

A pesar de la utilización, cada vez mayor, del abordaje endonasal directo transesfenoidal, en la resección de adenomas de hipófisis, existen pocos estudios que describan la frecuencia de complicaciones o molestias postoperatorias, relacionadas con este abordaje. En nuestro centro, comenzamos a emplear dicho abordaje desde Agosto del 2003, por lo que recolectamos la información de forma retrospectiva para describir la frecuencia de las complicaciones trans y postoperatorias, conjuntamente con la de las molestias postquirúrgicas relacionadas con este abordaje, en pacientes con adenoma de hipófisis.

\section{Pacientes y métodos}

Pacientes. Se realizó un muestreo no probabilístico de casos consecutivos, que incluyó pacientes mayores de 18 años, portadores de adenoma de hipófisis, en quienes se realizó el abordaje endonasal directo transesfenoidal, dentro de un periodo comprendido entre el 1 de Agosto del 2003 y el 31 de Diciembre del 2004, en la Unidad Médica de Alta Especialidad del Hospital de Especialidades del Centro Médico Nacional de Occidente. Se revisaron los expedientes clínicos de los pacientes seleccionados y se recolectó información sobre la presencia de complicaciones trans y postoperatorias, así como de las deformidades nasales estéticas y molestias postoperatorias, registradas en el expediente. Los datos faltantes, se completaron mediante una encuesta telefónica efectuada a cada paciente. Se excluyeron pacientes con adenoma de hipófisis operados anteriormente mediante otro abordaje y a quienes se les realizó dicho abordaje u otra ciru- gía endonasal por patologías distintas al adenoma de hipófisis. Un cirujano (J.M.P.O.) realizó todos los procedimientos.

Técnica quirúrgica. Se realizó el abordaje endonasal directo transesfenoidal, como fue descrito originalmente por Griffith y Veerapen ${ }^{10}$, con algunas modificaciones, como se describe a continuación. Se administraron antibióticos antes de la cirugía de manera profiláctica, después de la anestesia general, los pacientes se colocaron en posición supina, con la cabeza apoyada sobre un campo en forma de rosca para evitar la movilidad excesiva, elevando la cabeza hasta que el dorso nasal estuvo en paralelo con el piso, girando la cabeza $20^{\circ}$ hacia el hombro izquierdo. Se recortaron los pelos de la narina derecha, efectuando luego el aseo con jabón quirúrgico, seguido de la colocación de algodones humedecidos con descongestionante nasal por 5 minutos, se preparó el abdomen para una posible toma de injerto de grasa. En todos los casos se empleó la narina derecha y con ayuda del microscopio quirúrgico, además de fluoroscopia intermitente, se guió la introducción del rinoscopio hasta el cornete medio, infiltrando la mucosa nasal con solución inyectable, para facilitar la disección. Después se efectuó una incisión vertical en la mucosa del septum, a nivel de la unión del cartílago septal con el septum óseo, y se empujó el cartílago septal luxándolo hacia la izquierda, fuera de la línea media. Se identificó el vómer con ambos ostium esfenoidales a los lados y se retiró el rinoscopio, colocando en su lugar un separador de Hardy, a continuación el vómer fue retirado con ayuda de un cincel y una pinza de Kerrison, teniendo como límite superior ambos ostium esfenoidales. La mucosa del seno esfenoidal fue resecada, para retirar posteriormente el piso selar. La apertura de la duramadre fue a través de una incisión en cruz, procediendo luego a la resección del tumor con ayuda de cucharillas de anillo en bayoneta, pinzas de biopsia y succión. En un caso, se colocó injerto de grasa tomada de la pared abdominal. El vómer fue recolocado y se restituyó el cartílago septal hacia la línea media, además la mucosa nasal también fue reacomodada. Finalmente, se colocó empaquetamiento nasal bilateral y fue retirado tres días posteriormente. Los pacientes se egresaron del hospital tres a cinco días después de la cirugía.

\section{Resultados}

Se evaluaron en total 41 pacientes con adenoma de hipófisis, en quienes se empleó el abordaje endonasal directo transesfenoidal, con una relación hombre-mujer de 1.2:1 y una edad media de 47.7 años (Tabla 1). La hipertensión arterial se presentó en un $26.8 \%$ de la población, en tanto que un $65.9 \%$ no exhibieron enfermedades concurrentes (Tabla 1). 


\section{Tabla 1}

Características generales de la población

\begin{tabular}{lc}
\hline \multicolumn{1}{c}{$\mathrm{n}=41$} & Frecuencia (\%) \\
\hline Genero Hombre/Mujer & $23(56.1) / 18(43.9)$ \\
Edad (años) Desviación estándar & $47.7 \pm 16.8$ \\
Enfermedades concurrentes & \\
Ninguno (\%) & $27(65.9)$ \\
Hipertensión arterial & $11(26.8)$ \\
Diabetes mellitus tipo 2 & $4(9.8)$ \\
Infarto del miocardio & $2(4.9)$ \\
Evento vascular cerebral & $1(2.4)$ \\
Insuficiencia cardiaca & $1(2.4)$ \\
\hline
\end{tabular}

La complicación transoperatoria más frecuente fue la hemorragia intraselar en tres pacientes $(7.3 \%)$, seguida de hipertensión arterial en dos $(4.9 \%)$ y un caso con fístula de líquido cefalorraquídeo (2.4\%) (Figura 1).

Las complicaciones postoperatorias incluyeron diez casos (24.4\%) con diabetes insípida, en siete de los cuales fue transitoria y en tres permanente, por lo que requirieron sustitución hormonal. La insuficiencia hipofisaria anterior se presentó en dos casos que también precisaron reposición hormonal permanente. Un paciente tuvo sinusitis y no se presentaron deformidades nasales estéticas (perforación del septum nasal y deformidad nasal en silla de montar) o sinequias nasales (Figura 2).

Respecto a las molestias postoperatorias, la congestión nasal fue la más habitual con 13 casos (31.7\%), seguida por hiposmia en $6(14.6 \%)$ y sensación de flujo aéreo nasal disminuido en 4 (9.8) (Figura 3). Dos pacientes, en quienes no se reportaron complicaciones, murieron por causas no relacionadas a la cirugía, uno durante el primer mes de realizada la cirugía y el otro 6 meses después, ambos por infarto del miocardio.

\section{Discusión}

El abordaje transesfenoidal después de haber sido abandonado por Cushing, preservado por Dott y difundido por Guiot, en la actualidad, es la ruta preferida para alcanzar y remover diversas lesiones de la región selar $^{1,4,9,13-15,18}$.

Una de sus variantes, el abordaje endonasal directo transesfenoidal, descrito por Grifith y Veerapen en 1987, es empleado cada vez más, incluso en la cirugía transesfenoidal asistida por endoscopia ${ }^{3,7,10-12,20,21,23,27,28}$. Aún cuando esta variante se utiliza cada vez más, existen pocos estudios que mencionen la frecuencia y presencia de complicaciones asociadas a su uso.

Los estudios existentes, dentro de las complicaciones transoperatorias muestran porcentajes de fístula de líquido cefalorraquídeo entre 1 y 4.8\% ${ }^{6,19,28}$. Nuestro estudio mostró un caso $(2.4 \%)$ con fístula de líquido cefalorraquídeo, el

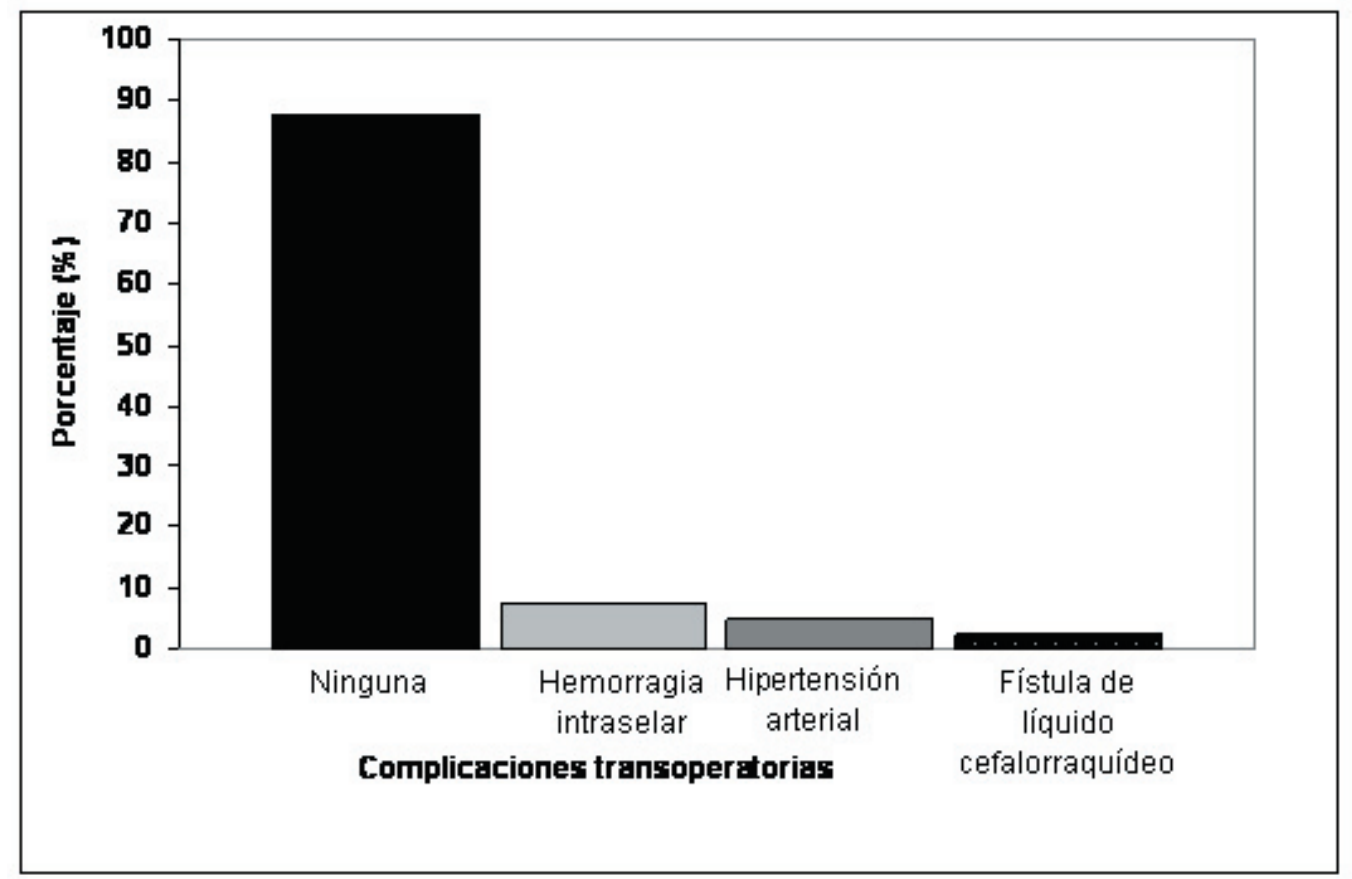

Figura 1. Porcentaje de complicaciones transoperatorias. 


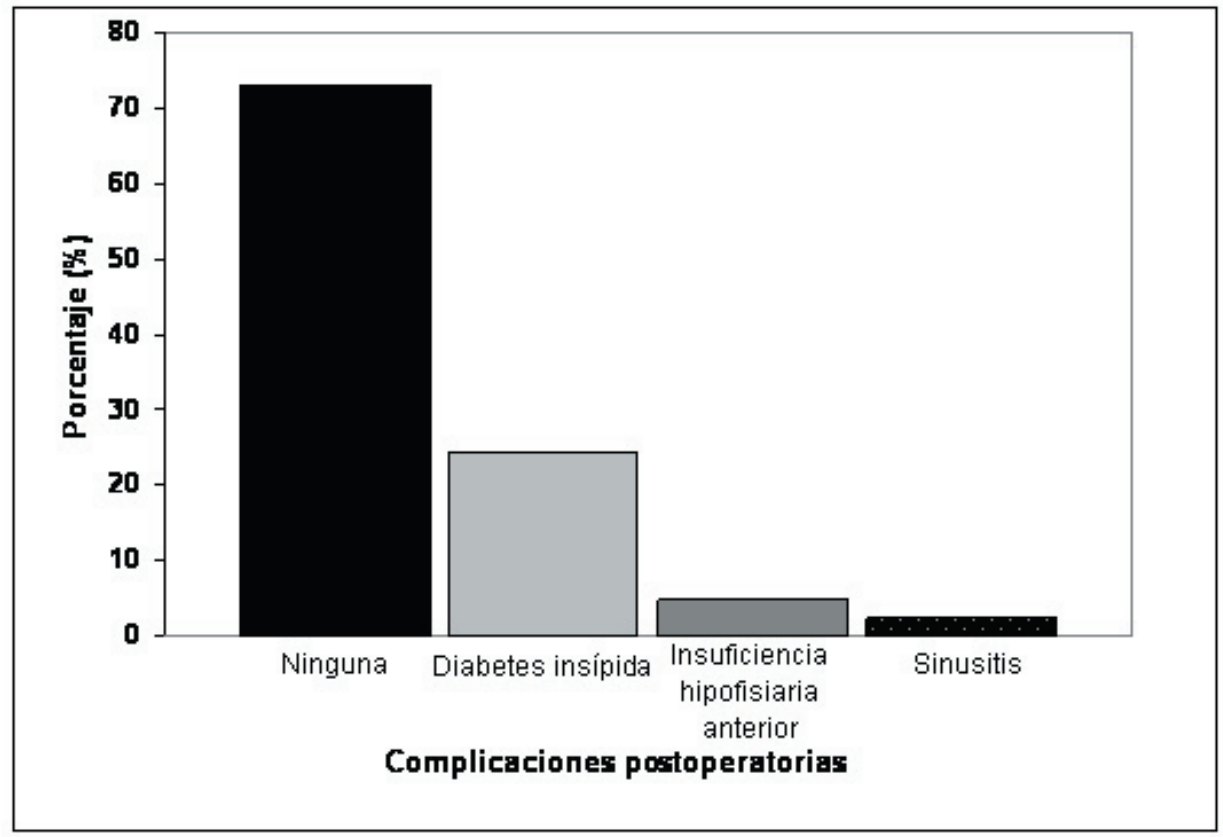

Figura 2. Porcentaje de complicaciones postoperatorias.

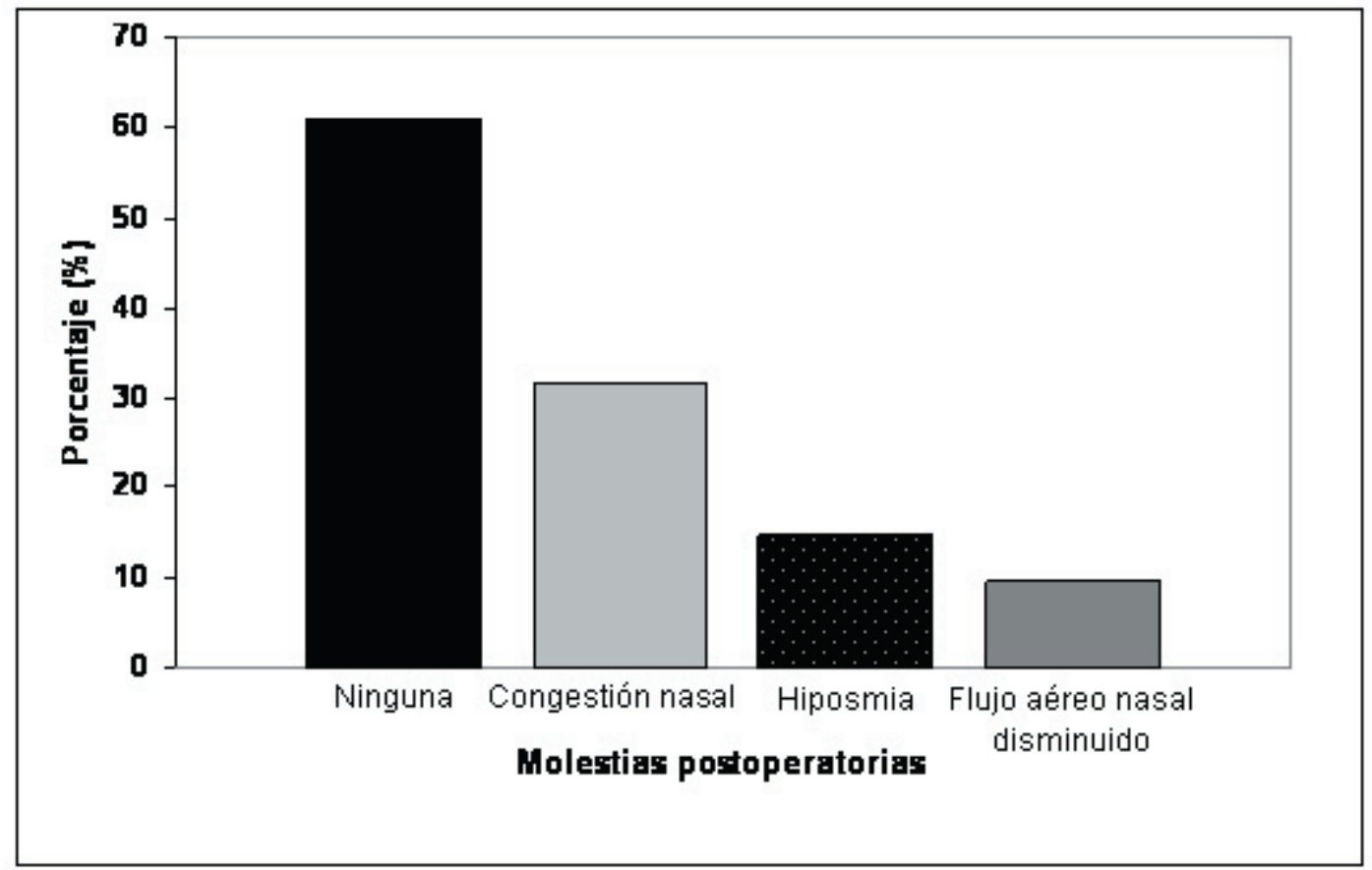

Figura 3. Porcentaje de molestias referidas por el paciente.

cual se identificó durante la cirugía, colocándosele por ello, injerto de grasa tomada de la pared abdominal, conjuntamente con matriz de colágeno y drenaje lumbar durante 5 días, sin requerir manejo ulterior.

Otra complicación transoperatoria, la hemorragia intraselar, que la definimos como la presencia postoperatoria de sangre confinada a la silla turca, varía del 0.4 al 3\% y se ha relacionado con la presencia de tumor residual, hemostasia inadecuada y entrada al seno cavernoso, entre otras causas ${ }^{6,24}$. Nuestro estudio reveló tres casos de hemorragia intraselar con tumor residual, que no pudo ser resecado en su totalidad mediante el abordaje endonasal 
directo transesfenoidal, debido a la consistencia firme del tumor. El primer caso fue una mujer de 81 años, con hipertensión arterial de difícil control; el segundo fue un hombre de 76 , años quien después de la cirugía tuvo incremento de la presión arterial con sangrado y el tercero, un paciente masculino de 31 años con adenoma productor de hormona del crecimiento e hipertensión arterial. Los tres pacientes, que inicialmente tenían hemianopsia bitemporal, presentaron empeoramiento de la agudeza visual súbito hasta visión de bultos y hubo que reoperarlos mediante un abordaje pterional transcraneal, para lograr la resección total. Durante la reintervención, se encontró sangrado del tumor residual, que cedió una vez resecado el tumor; los tres pacientes recuperaron la agudeza visual que se normalizó 3 meses después. Por lo anterior, es importante destacar que la selección de pacientes, para un abordaje endonasal transesfenoidal, debe ser minuciosamente cuidada, tratando de identificar características tales como: consistencia firme del tumor, vascularidad, posición de las arterias carótidas internas, entre otras, que puedan predisponer a complicaciones como la hemorragia intraselar.

En relación a las complicaciones anestésicas, una encuesta nacional expuso una prevalencia del $2.8 \%$, sin embargo, hasta el $10 \%$ de los cirujanos encuestados atestiguaron una complicación anestésica, sin aclarar el tipo, manejo y duración de esta ${ }^{6}$. Por otra parte, en nuestra serie únicamente encontramos elevación de la presión arterial durante la cirugía, que ameritó la suspensión transitoria de ésta, debido a que el sangrado impedía la visibilidad. Dicha elevación, finalmente fue controlada y la cirugía continuó sin otra complicación. No hubo epistaxis, ni lesiones de la arteria carótida interna o alguna otra complicación transoperatoria registrada.

Dentro de las complicaciones postoperatorias, las alteraciones endocrinas son las más frecuentes. Estudios previos muestran que del 10 al $60 \%$ de los postoperados desarrollan diabetes insípida transitoria (aquélla que dura entre 12 y 36 horas después de la cirugía) y del 0.5 al $15 \%$ es permanente. En nuestro estudio hubo $17.1 \%$ casos con diabetes insípida transitoria y en $7.3 \%$ ésta fue permanente, en ambos casos dentro del rango antes mencionado. Por otra parte, la insuficiencia hipofisaria anterior se ha descrito hasta en el $10 \%$ de los casos y en nuestra serie fue de $4.9 \%$.

Ciric y cols, mencionan que la sinusitis se presenta en aproximadamente $8.5 \%$, de los operados vía endonasal. En nuestra investigación sólo hubo un caso, el cual tenía sinusitis antes de la cirugía y mejoró antes de la resección. Sin embargo, después del procedimiento quirúrgico la desarrolló nuevamente, requiriendo tratamiento antibiótico. Finalmente el paciente se recuperó totalmente. A diferencia de otras series, en la nuestra no encontramos sinequias o hipoestesia en el labio superior. Esta última, se ha descrito habitualmente en el abordaje sublabial transesfenoidal. Además, tampoco encontramos deformidades nasales estéticas, que son frecuentes en el abordaje endonasal transeptal transesfenoidal, una de estas, la perforación septal, tiene una frecuencia que fluctúa entre 0.3 y $3 \%$.

Las molestias nasales postoperatorias poco se han tomado en cuenta, el único reporte encontrado es el referido por Zada y cols. ${ }^{28}$, en donde encuestaron a 78 pacientes, de los cuales el $60 \%$ se quejaron de congestión nasal, $52 \%$ de flujo aéreo disminuido y $53 \%$ de hiposmia en diversos grados. Por otra parte, en nuestra serie la prevalencia de molestias son considerablemente menores, la más frecuente, es la congestión nasal que no duró más de 3 semanas; seguida de hiposmia transitoria y flujo aéreo nasal disminuido, una explicación para estas molestias podría estar relacionada con el empaquetamiento nasal, puesto que todos los pacientes se quejaban de no poder respirar a través de la nariz o de tener que hacerlo por la boca.

Las limitantes de nuestro trabajo son las esperadas de los estudios retrospectivos, por lo que no se puede afirmar categóricamente las ventajas de este abordaje. Pese a lo anterior, esta investigación mostró que el abordaje endonasal directo transesfenoidal tiende a producir menos complicaciones y molestias, sobre todo de tipo nasal, lo cual probablemente se explica porque se realiza una menor disección de la mucosa nasal. Es importante tener en cuenta la presencia de molestias nasales ya que se les presta poca atención por no amenazar la vida, no obstante, afectan la comodidad del paciente y podrían disminuirse evitando el empaquetamiento nasal.

En conclusión, el abordaje endonasal directo transesfenoidal tiende a presentar menos complicaciones, especialmente de tipo nasal (hipoestesia del labio superior, perforación del septum y deformidad nasal en silla de montar), con disminución de las molestias nasales, por lo que en el futuro podría considerarse el abordaje preferido para la resección de adenomas de hipófisis.

\section{Bibliografía}

1. Abosch, A., Tyrrell, J.B., Lamborn, K.R., et al.: Transsphenoidal microsurgery for growth hormone-secreting pituitary adenomas: initial outcomes and long term results. J Clin Endocrinol Metab 1998; 83: 3411-3418.

2. Asa, S.L., Shereen, E.: The Classification of Pituitary Tumors: An Update. Seminars in Neurosurgery 2001; 12: 273288.

3. Badie, B., Nguyen, P., Preston, J.K.: Endoscopic-guided direct endonasal approach for pituitary surgery. Surg Neurol 2000; 53: 168-173.

4. Blevins, L.S. Jr, Chrity, J.H., Khajavi, M., et al.: Out- 
comes of therapy for Cushing's disease due to adrenocorticotropin-secreting pituitary macroadenomas. J Clin Endocrinol Metab 1998, 83: 63-67.

5. Cawley, M., Tindall, G.T.; Transsphenoidal Surgery: Operative Techniques; In Krisht AF, Tindall GT (ed). Pituitary Disorders. Lippincott Williams \& Wilkins, 1999; pp. 349359.

6. Ciric, I., Ragin, A., Baumgartner, C.: Complications of transsphenoidal surgery: results of a national survey, review of the literature, and personal experience. Neurosurgery 1997; 40: 225-237.

7. Cooke, R.S., Jones, R.A.C.: Experience with the direct transnasal transsphenoidal approach to the pituitary fossa. Br J Neurosurg 1994; 8: 193-196.

8. Das, K., Spencer, W., Nwagwu, C.I., Schaeffer, S., Wenk, E., Weiss, M.H., Couldwell, W.T.: Approaches to the sellar an parasellar region: anatomic comparison of endonasaltranssphenoidal, sublabial-transsphenoidal, and transethmoidal approaches. Neurol Res 2001; 23: 51-54.

9. Fahlbusch, R., Honegger, J., Paulus, W., et al.: Surgical treatment of craniopharyngiomas: experience with 168 patients. J Neurosurg 1999; 90: 237-250.

10. Griffith, H.B., Veerapen, R.: A direct transnasal approach to the sphenoid sinus. Technical note. J Neurosurg 1987; 66: 140-142.

11. Kawamata, T., Iseki, H., Ishizaki, R., et al.: Minimally invasive endoscope-assisted endonasal trans-sphenoidal microsurgery for pituitary tumors: experience with 215 cases comparing with sublabial trans-sphenoidal approach. Neurol Res 2002; 24: 259-265.

12. Koren, I., Hadar, T., Rappaport, Z.H., et al.: Endoscopic transnasal transsphenoidal microsurgery versus the sublabial approach for the treatment of pituitary tumors: endonasal complications. Laryngoscope 1999; 109: 1838-1840.

13. Lanzino, G., Laws, E.R. Jr.: Pioneers in the development of transsphenoidal surgery: Theodor Kocher, Oskar Hirsch, and Norman Dott. J Neurosurg 2001; 95: 1097-1103.

14. Lanzino, G., Laws, E.R. Jr.: Key personalities in the development and popularization of the trnassphenoidal approach to pituitary tumors: and historical review. Neurosurg Clin N Am 2003; 14: 1-10.

15. Laws, E.R. Jr.: Transsphenoidal approach in the management of craniopharyngioma. J. Neurosurg 1980; 52: 661-666.

16. Lee, M.: Medical treatment of functional pituitary tumors. Neurosurg Clin N Am 2003; 14: 81-87.

\section{Comentario al trabajo: Complicaciones del abordaje endonasal directo transesfenoidal en el manejo de adenomas de hipófisis de J.H. Sandoval-Sánchez y cols}

Los autores revisan su experiencia en el tratamiento por vía endonasal de 41 adenomas hipofisarios. El artículo hace especial énfasis en las complicaciones relacionadas con el
17. Liu, J.K., Das, K., Weiss, M.H.: The history and evolution of transsphenoidal surgery. J Neurosurg 2001; 95:1083-1096.

18. Liu, J.K., Wiess, M.H., Couldwell, W.T.: Surgical approaches to pituitary tumors. Neurosurg Clin N Am 2003; 14: 93-107.

19. Lopez-Arbolay, O., Morales-Sabina, O., GonzálezGonzález, J.L., Valdés-Lorenzo, N.: Transsphenoidal approach to prolactinomas. Neurocirugía 2006; 17: 226-231.

20. Nasseri, S.S., Kasperbauer, J.L., Strome, S.E., et al.: Endoscopic transnasal pituitary surgery: report on 180 cases. Am J Rhinol 2001; 15: 281-287.

21. Rhoton, A.L.: The Sellar Region. Neurosurgery 2002 51 (suppl 1): 335-374.

22. Sanno, N., Teramoto, A., Osamura, Y.: Pathology of pituitary tumors. Neurosurg Clin N Am 2003; 14: 25-39.

23. Sheehan, M.T., Atkinson, J.L., Kasperbauer, J.L., et al.: Preliminary comparison of the endoscopic transnasal vs the sublabial transseptal approach for clinically nonfunctioning pituitary macroadenomas. Mayo Clin Proc 199; 74: 661-670.

24. Tindall, G.T., Woodard, E.J., Barrow, D.L.: Pituitary adenomas; In Apuzzo MLJ (ed). Brain Surgery Complication Avoidance and Management; Churchill Livingstone, 1993; pp. 269-312.

25. Vrionis, F.D., Saatman, D., Sorenson, J., Brem, S.: Microscopic paraseptal sphenoidotomy approach for pituitary tumors. Cancer C. 2002; 9: 223-231.

26. Weiss, M.H.: Transnasal transsphenoidal approach. In Apuzo MLJ(ed): Surgery of the third ventricle. Williams \& Wilkins, Baltimore 1987: 476-494.

27. White, W.L., Lanzino, G., Feiz-Erfan, I.: The transseptal transsphenoidal approach to pituitary tumors. Barrow Quarterly 2002; 18: 20-28.

28. Zada, G., Kelly, D.F., Cohan, P.: Endonasal transsphenoidal approach for pituitary adenomas and other sellar lesions: an assessment of efficacy, safety, and patient impressions. J Neurosurg 2003; 98: 335-338.

Sandoval-Sánchez, J.H.; Flores-Cárdenas, F.; VargasFrutos, M ${ }^{\mathrm{a}}$.C.; Páez-Ontiveros, J.M.: Complicaciones del abordaje endonasal directo transesfenoidal en el manejo de adenomas de hipófisis. Neurocirugía 2007; 18: 485-491.

Correspondencia postal: José Humberto Sandoval Sánchez. Calle: Isaac Albeniz \# 3847. Colonia Insurgentes. Guadalajara Jalisco, México. CP 44820.

abordaje transnasal recomendando una variante del mismo, con incisión del septum más posterior que la habitual, en la unión del tabique cartilaginoso y el óseo y que en su expe- 
riencia produce menos complicaciones intranasales. Las complicaciones recogidas, referidas a la cirugía hipofisaria propiamente dicha son similares a las recogidas en revisiones amplias de cirugía hipofisaria ${ }^{1}$.

En estos tiempos, en que se extienden cada vez más las técnicas endoscópicas de cirugía hipofisaria, sin tiempo nasal propiamente dicho, que eliminan las complicaciones nasales del abordaje hipofisario ${ }^{2}$, y mientras no empleamos de forma generalizada la endoscopia, la variante empleada por los autores puede ayudarnos a disminuir las complicaciones nasales de la cirugía selar "convencional".

\section{Bibliografía}

1. Ciric, I., Ragin, A., Baumgartner, C.: Complications of transsphenoidal surgery: results of a national survey, review of the literature, and personal experience. Neurosurgery 1997; 40: 225-237.

2. Kawamata, T., Iseki, H., Ishizaki, R., Hori, T.: Minimally invasive endoscope-assisted endonasal trans-sphenoidal microsurgery for pituitary tumors: experience with 215 cases comparing with sublabial trans-sphenoidal approach. Neurol Res. 2002 ; 24: 259-265.

C. Conde Alonso 